\title{
A Sperm Testing Device on a Liquid Crystal and Polymer Composite Film
} Yi-Hsin Lin ${ }^{1 *}$, Ting-Yu Chu ${ }^{1}$, Wei-Lin Chu ${ }^{1}$, Yu-Shih Tsou ${ }^{1}$, Ya-Ping Chiu ${ }^{2}$, Farn Lu ${ }^{3}$ Wan-Chen Tsai ${ }^{3}$ and Shin-Tson Wu ${ }^{4}$

${ }^{1}$ Department of Photonics, National Chiao Tung University, Hsinchu, Taiwan, R.O.C

${ }^{2}$ Department of Physics, National Sun Yat-Sen University, Kaohsiung, Taiwan, R.O.C

${ }^{3}$ Department of Obstetrics and Gynecology, Ton Yen General Hospital, Hsinchu, Taiwan, R.O.C

${ }^{4}$ College of Optics and Photonics, University of Central Florida, Orlando, Florida, USA

\begin{abstract}
Developing a handy sperm testing device is important since sperm quality is a significant factor for fertility potential. In this paper, we demonstrate a sperm testing device based on a switchable surface, a liquid crystal and polymer composite film (LCPCF). The wettability of LCPCF is electrically switchable due to the electrically tunable orientations of liquid crystal molecules. In experiments, two motions of a semen drop on switchable surface of LCPCF are observed: back-and-forth stretches and collapses of semen drops. The better quality spermatozoa results in back-and-forth stretches of a semen drop on LCPCF; otherwise, the semen drop collapses. The motility and concentration of semen can also be sensed by the stretch distance and collapse distance of semen drops, respectively. The mechanism of back-and-forth stretches of semen drops results from fertile sperms swimming against the flow with the periodic changes of the orientation of LC molecules with pulsed voltages. The mechanism of collapses of semen drops results from the washed-away infertile sperms which are deposited on LCPCF and then re-modify surface of LCPCF. Potential applications for this device include sperm testers and microfluidic devices for Assisted Reproductive Technology.
\end{abstract}

Keywords: Liquid crystal; LC; Sperm testing; Switchable surface

\section{Introduction}

Infertility, a global human issue, is primarily governed by the quality of human spermatozoa or sperms, such as concentration, motility, and morphology. Most males are reluctant to have their sperm tested in hospitals due to cost and discomfort. It is highly desirable to develop a handy sperm testing device to be used in the comfort of one's home. Our motivation is to realize a sperm testing device based on the droplet manipulation on a switchable surface. Conventionally, switchable surfaces utilize changes in molecular conformation of a selfassembled monolayer (SAM) under external stimuli [1-11]. However, a fluidic drop usually refuses to move toward more hydrophilic regions on the SAM-based switchable surfaces because the weak chemical gradient cannot overcome the hysteresis of the surface. Moreover, the fabrication of SAM is complicated and costly, especially for large areas. Recently, we have developed a switchable-nanostructured surface based on the orientations of liquid crystal (LC) molecules anchored among the polymer grains on the liquid crystal and polymer composite film (LCPCF) which is a result of photo-induced phase separation between the LC and the polymer [12,13]. The wettability of LCPCF is electrically tunable because of different wetting properties between the phenyl rings and the terminal group (cyano) of LC molecules whose orientations could be switchable by applied electric fields. The droplet is manipulated on LCPCF with a wettability gradient and can be designed for the applications of polarizer-free electro-optical switches [14]. Up to now, no report has been found concerning the development of sperm tester using switchable surfaces. In this paper, we demonstrate a sperm testing device based on the semen droplet manipulation on LCPCF. By either a back-and-forth stretch or a collapse of a semen droplet on LCPCF within periodically switched LC molecular reorientation, LCPCF can sense the semen through the motion of a semen droplet which in turn depends on the information of spermatozoa in the semen. The potential application of sperm testing devices is in Assisted Reproductive Technology.

\section{Materials and Methods}

\section{Structure Of a Sperm Testing Device and Sample Preparation of LCPCF}

The structure of a sperm testing device based on LCPCF is illustrated in Figures $1 \mathrm{~A}$ and $1 \mathrm{~B}$. The structure consists of a LCPCF on a patterned indium tin oxide (ITO) glass substrate to provide fringe electric fields at the surface. The ITO electrodes on the glass substrate were etched with interdigitated chevron patterns, shown as the zigzag electrodes in the Figure 1A. The zigzag ITO strips have corner angles of $150^{\circ}$. The width and gap of the electrode strips are $4 \mu \mathrm{m}$ and $14 \mu \mathrm{m}$, respectively. In order to manipulate a semen drop on LCPCF, the two regions of the interdigitated chevron electrodes were patterned identically, as shown in Figure 1A. The distance between two regions is $20 \mu \mathrm{m}$ and the width of each region is $2 \mathrm{~mm}$. At null voltage $(\mathrm{V}=0)$, the contact angles on both sides of the droplet are the same. When we apply a voltage to the left interdigitated region (the red region), the left region of LCPCF is more hydrophilic because of the tilts of LC molecules anchored to the polymer grains, so the droplet experiences a net Young's force [14-16] and is forced to move toward the left. The magnification of the LCPCF surface including polymer grains (purple) and liquid crystals (yellow dots) among polymer grains in Figure $1 \mathrm{~A}$ is exaggeratedly illustrated in Figure 1(B). At $\mathrm{V}=0$, the rod-like LC molecules are aligned along $\mathrm{y}$-direction. Under an applied alternating current $(\mathrm{AC})$ voltage $(f=1$

*Corresponding author: Yi-Hsin Lin, Department of Photonics, National Chiao Tung University, Hsinchu, Taiwan, R.O.C, Tel: +886-3-5712121: Fax: +886-35735601; E-mail: yilin@mail.nctu.edu.tw

Received December 01, 2011; Accepted December 17, 2011; Published December 19, 2011

Citation: Lin Y, Chu T, Chu W, Tsou Y, Chiu Y, et al. (2011) A Sperm Testing Device on a Liquid Crystal and Polymer Composite Film. J Nanomedic Nanotechnol S9:001. doi:10.4172/2157-7439.S9-001

Copyright: (c) 2011 Lin Y, et al. This is an open-access article distributed unde the terms of the Creative Commons Attribution License, which permits unrestricted use, distribution, and reproduction in any medium, provided the original author and source are credited. 
$\mathrm{kHz}$ ), the LC molecules are reoriented along the electric fields. The LCPCF is more hydrophobic at $0 \mathrm{~V}_{\mathrm{rms}}$ because the phenyl rings of the E7 LC molecules are more parallel to the surface of the LCPCF ( $x-y$ plane). On the other hand, the LCPCF is more hydrophilic in a high voltage state because of the field-induced uneven tilts on the cyano terminal groups of the E7 LC molecules near the edges of the fringe electric fields. Moreover, the hydrophile of the LCPCF is electrically tunable by changing the magnitude of the voltage.

To fabricate LCPCF on the ITO glass substrate, we mixed a nematic LC mixture E7 (Merck) and a liquid crystalline monomer (4-(3-Acryloyloxypropyloxy)-benzoic acid 2- methyl-1, 4-phenylene ester) at 70:30 wt \% ratios. The mixtures were then filled into an empty cell with a gap of $12 \mu \mathrm{m}$ which consists of a glass top substrate and a patterned ITO bottom glass substrate. The top substrate of the cell was overcoated with a thin polyimide (PI) layer and then mechanically buffed at the direction of $20^{\circ}$ with respect to the electrode strips. After filling, the cell was exposed to a UV light with intensity $I=10 \mathrm{~mW} / \mathrm{cm}^{2}$ for $\sim 30 \mathrm{~min}$ at $70^{\circ} \mathrm{C}$. After phase separation and photo-polymerization, the top glass substrate was peeled off by a thermal-releasing process. A solidified LCPCF was obtained with $12 \mu \mathrm{m}$ thickness and $30 \mathrm{~nm}$ rootmean-squared roughnesses.

To observe the motion of a semen drop on LCPCF, we dripped a semen drop on LCPCF under inhomogeneous distribution of LC reorientations induced by fringing electric fields. A semen drop consisted of seminal plasma and spermatozoa (or sperms). We recorded the dynamics of the semen drops by a CCD camera (JAI CV-M30) with a frame rate of 360 fields/sec and measured the contact angles of the semen droplet with time by contact angle measurement (FTA 1000 Analyzer System). A semen drop of $3 \mu \mathrm{l}$ was laid on the LCPCF surface. We then applied $150 \mathrm{~V}_{\text {m }}$ square pulses $(f=1 \mathrm{kHz})$ to the left electrodes shown in Figure $1 \mathrm{~A}$ for a time duration of 500 ms. The semen samples are donated by 31 males between 26 and 45 years old. When we recorded the dynamics of semen drop, stretches of semen drops and collapses of semen drops were observed. The distance difference of the left contact angle at $0.5 \mathrm{sec}$ and at $0 \mathrm{sec}$ for stretches of semen drops is defined as stretch distance. The distance difference of the left contact angle at $0.5 \mathrm{sec}$ and at $0 \mathrm{sec}$ for collapses of semen drops is defined as collapse distance.

\section{Results and Discussions}

Figure 2 shows the SEM (Scanning Electron Microscopic) image of the surface of LCPCF after we removed the LC by hexane. The image was taken by using a Scanning Electron Microscope (JEOL JSM- 7401F FE-SEM). The size of the LC domains is around 100-200 $\mathrm{nm}$ and the size of polymer grains is around $50 \mathrm{~nm}$. The root-meansquare (RMS) roughness of the film surfaces was measured to be $\sim 30$ $\mathrm{nm}$ by an atomic force microscope (AFM) (Dimension 3100, Digital Instruments). On the LCPCF, the LC molecules were anchored among the polymer grains along the direction parallel to the rubbing direction of the PI layer (white arrow) which can be confirmed by observing the transmission of the LCPCF between two crossed polarizers.

A semen drop of $3 \mu \mathrm{l}$ was laid on the LCPCF and then applied 150 $\mathrm{V}_{\text {rms }}$ square pulses $(f=1 \mathrm{kHz})$ to the left electrodes, as shown in Figure $1 \mathrm{~A}$ for a time duration of $500 \mathrm{~ms}$. Among all samples, two motions of semen droplets were observed: back-and-forth stretch [Figure 3A] and collapse [Figure 3B]. Here, the back-and-forth stretch of a droplet means the contact angles of a droplet change periodically with pulsed voltages, but the droplet stays in the same location. The collapse of a droplet means the contact angle changes immediately when we turn on the voltage and then the contact angle does not recover after we turn off the voltage. In Figure 3A, the contact angles on the right and on the left were around $69^{\circ}$ at voltage-off state at $0 \mathrm{sec}$. At $0.5 \mathrm{sec}$, the voltage was then applied on the left region (the left of the dotted white line in Figure 3A), the left region of the semen drop was more hydrophilic and the contact angle on the left $\left(\sim 50.3^{\circ}\right)$ decreased due

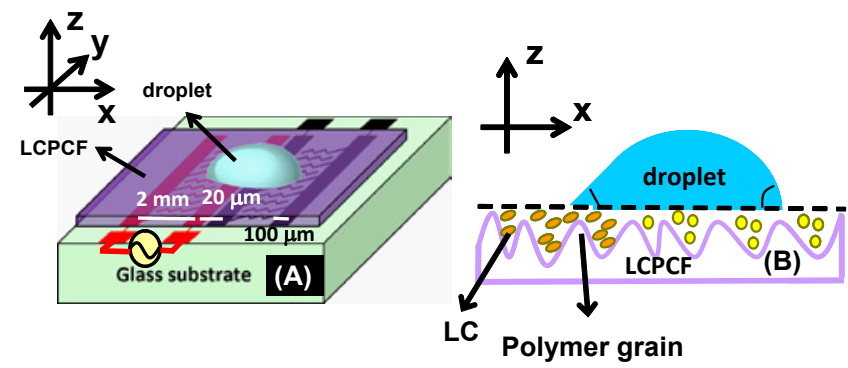

Figure 1: (A) Schematic of the structure of LCPCF, (B) the magnification of the surface of LCPCF. At voltage-off, rod-like LC molecules anchored among the polymer grains are aligned along y-direction. (Yellow dots). At voltage-on states, LC molecules tilt up by the electric fields. (Orange elliptical dots). The tilted LC molecules result in more hydrophilic surface properties of LCPCF.

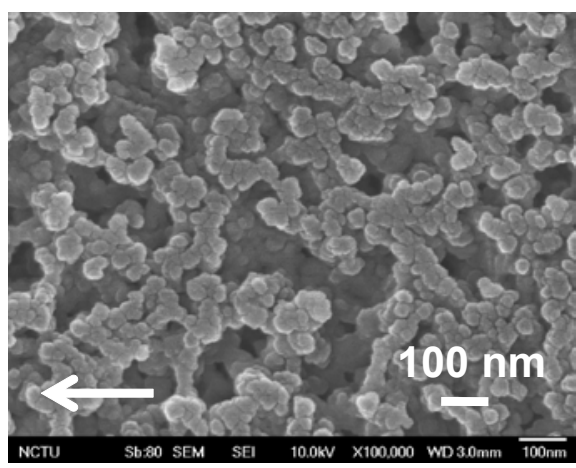

Figure 2: The SEM image of LCPCF. The arrow indicates the rubbing direction of PI layer before peeling off the top substrate.
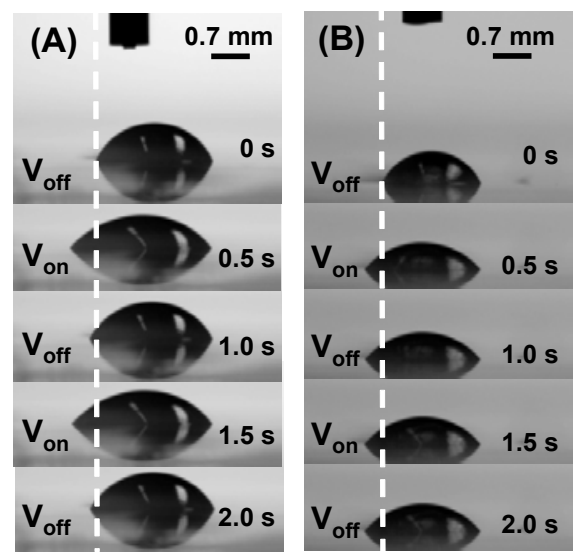

Figure 3: (A) The stretch of a semen drop at different time. The left side of the semen drop stretches when the voltage is on and off. (B) The collapse of a semen drop at different time. The left side of the semen drop collapses when the voltage is on and off. The voltage was applied in the region left to white dotted line. 
to the orientations of LC molecules on the surface. Because of the high viscosity of semen drop; as a result, the net forces of the semen drop are inadequate to move the semen droplet forward even though the surface of LCPCF has a wettability gradient. The left side of the semen drop then pulled the right side of the semen drop and the contact angle on the right side also decreased $\left(\sim 55.9^{\circ}\right)$. When the voltage was turned off at $1 \mathrm{sec}$, the left contact angle recovered to the high contact angle $\sim 63.0^{\circ}$. When we applied voltages periodically, we observed the backand-forth stretch of the semen drop (Figure 3A). The recovered contact angle (i.e. contact angle at $\mathrm{V}_{\text {off }}$ ) decreases gradually when the voltage is on and off many times. Figure 3B shows the collapse of the semen drop. Similarly, the left contact angle $\left(\sim 51^{\circ}\right)$ is smaller than the other $\left(\sim 54^{\circ}\right)$ when we applied voltage on the left region at $0.5 \mathrm{sec}$. After that, the contact angles did not change when we applied voltages periodically. The semen drop collapsed with the pulsed voltages.

In order to understand the mechanism of the collapse and backand-forth stretch of semen drops, we also observed the dynamics of a semen droplet under an inverted optical microscopy (Leica DMI3000B). We captured 4 images at different times as shown in Figures $4 \mathrm{~A}$ to $4 \mathrm{D}$, circling three sperms in blue (blue sperms) and two sperms in red (red sperms). After the voltage was turned on at $4.5 \mathrm{sec}$, the semen droplet moved toward more hydrophilic region in the left part of Figures $4 \mathrm{~A}$ to $4 \mathrm{D}$. The induced flow of seminal plasma went toward the left and then washed away the blue and red sperms. However, the red sperms swim upstream against the flow of seminal plasma. The selfmovement of sperm against flow direction has been reported [17]. As to the collapse of the semen drop, the similar wash-away sperms were observed when the voltage was on and we did not observe the sperms swim against the fluidic flow. We then observed that dried surface of LCPCF under a microscope after performing the collapse of the semen droplet, as shown in Figure 5. Most of the sperms aggregated in the applied voltage region in Figure 5. The collapse of the semen drop results from the deposition of the sperms on LCPCF which re-modifies the surface of LCPCF. Therefore, the conditions of sperms affect the motions of semen drop on LCPCF.

The two observed motions of semen drops on LCPCF are then compared with the parameters of standard semen analysis in the hospital, such as concentration, motility, and morphology. Motility is the ratio of motile sperms to total sperm number, grade A defined as the percentage of spermatozoa swimming in a forward direction, and grade $\mathrm{B}$ defined as the percentage of sperms moving in circles. Morphology indicates the percentage of normal sperm heads. According to World Health Organization (WHO) guidelines, a standard sperm analysis is performed for measuring the parameters of spermatozoa $[18,19]$. The concentration, motility, grade A and grade B were counted with a Makler counting chamber (Irvine Scientific). The Spermac stain method (sps250, FertiPro) was used for assessing sperm morphology. Figures $6 \mathrm{~A}, 6 \mathrm{~B}, 6 \mathrm{C}$ and $6 \mathrm{D}$ are the motility, morphology, grade $\mathrm{A}$, and grade $\mathrm{B}$ as a function of the semen concentration for the collapse (black squares) and the stretch (blue dots) of semen droplets. From Figures $6 \mathrm{~A}, 6 \mathrm{~B}, 6 \mathrm{C}$ and $6 \mathrm{D}$, the semen drop stretches back and forth only when the semen concentration is larger than $\sim 100$ million/mL, morphology is larger than $\sim 15 \%$, motility is larger than $\sim 50 \%$, grade $\mathrm{A}$ is larger than $\sim 30 \%$, and grade $B$ is less than $\sim 30 \%$. Otherwise, the semen droplet collapses. Higher concentration and motility of a man's semen increase chances for conception. The high grade A and low grade B semen translates to more motile spermatozoa moving in a straight line with good speeds, which is preferred for natural conception. The high morphology of semen means there are fewer abnormal spermatozoa, and so the likelihood of infertility is decreased. Therefore, the stretch

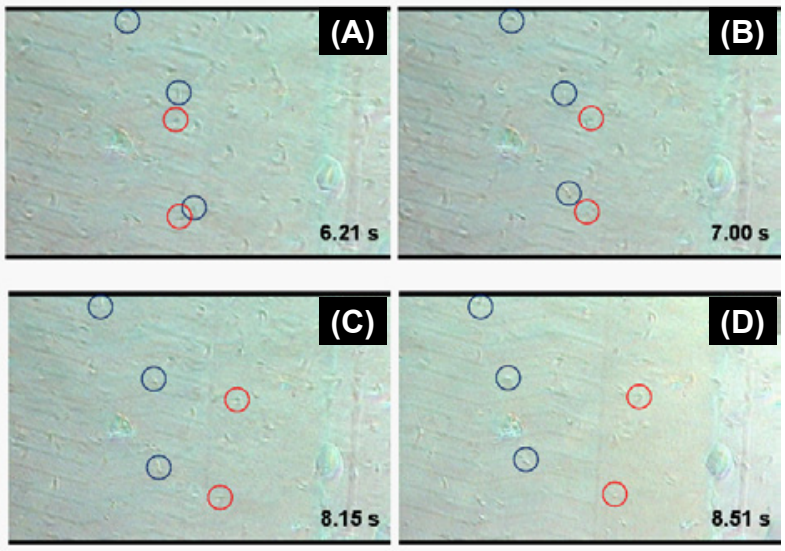

Figure 4: The microscopic images of a semen droplet at (A) $6.21 \mathrm{sec},(B) 7$ $\mathrm{sec},(C) 8.15 \mathrm{sec}$ and (D) $8.51 \mathrm{sec}$. The voltage was applied at $4.5 \mathrm{sec}$ on the left region of the images. We circled representative infertile sperms by blue circles and two fertile sperms by red circles. The induced flow of seminal plasma went to the left and then washed away the infertile sperms (blue circles). The fertile sperms (red circles) swam against the flow of seminal plasma.

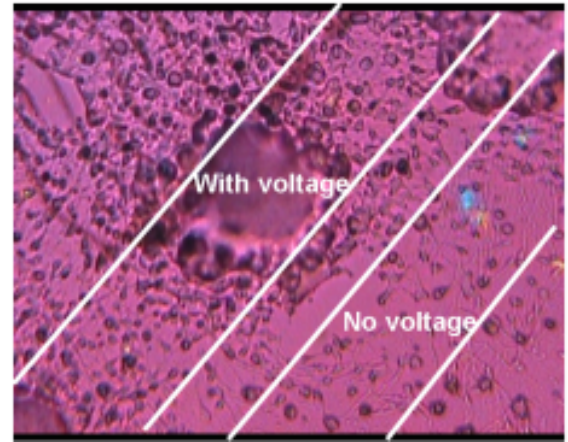

Figure 5: The dried surface of LCPCF after performing the collapse of the semen droplet under a microscope. Most of spermatozoa gather together on the region with applied voltage.

or collapse of a semen drop serves the purpose of probing the quality of spermatozoa. The stretch of a semen drop is a result of better quality semen for human reproductions while the collapse of a semen drop shows the contrary.

Comparing the sperm parameters with the stretching distance and the collapse distance, we found that the stretch distance of the stretching semen drops has linear relation with motility, as shown in Figure 7A. The stretch distance increases from $0.115 \mathrm{~mm}$ to 0.390 mm with an increase of motility from $50 \%$ to $90 \%$. When motility is less than $50 \%$, the semen drops do not have stretching motions. As a result, the stretch of semen drop can not only indicate better quality of semen, but also indicate how much the motility is. The collapse distance of collapsing semen drops as a function of concentration of semen drops is also shown in Figure 7B. The collapse distance is around $0.2-0.5 \mathrm{~mm}$ when the concentration of collapsing semen drops is around 50-100 million/mL. Therefore, we can test the quality semen by the two motions of the semen on LCPCF and also sense the motility or concentration according to the stretch distance or collapse distance.

Based on the experimental results, the mechanisms of the back-andforth stretch (Figure 8A, 8B, and 8C) and the collapse (Figures 8D, 8E, 

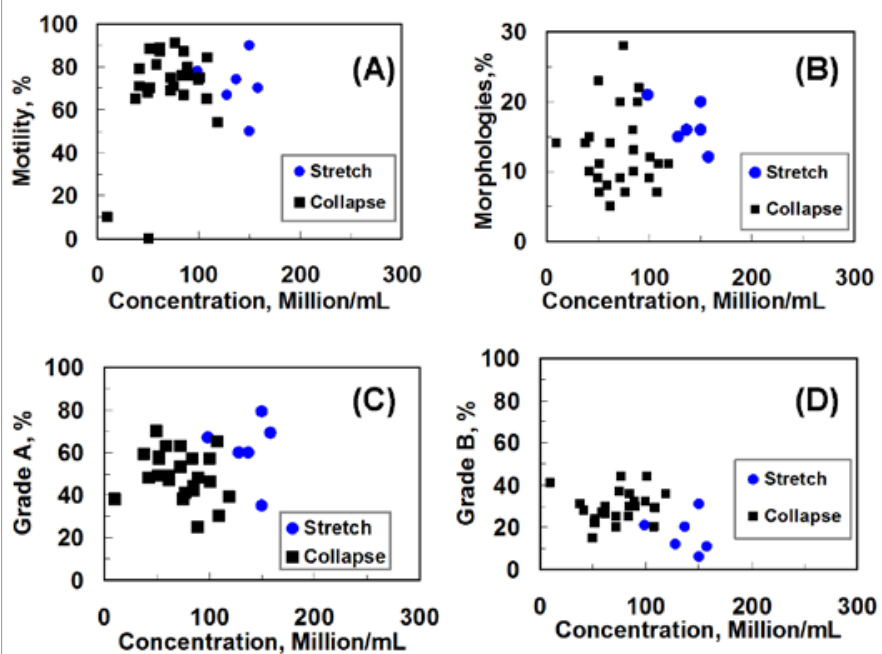

Figure 6: (A) Motility, (B) morphology, (C) grade A and (D) grade $B$ as a function of concentration. Blue dots stand for stretching motion of the semen droplet and black squares stand for collapsing motion of the semen droplet.
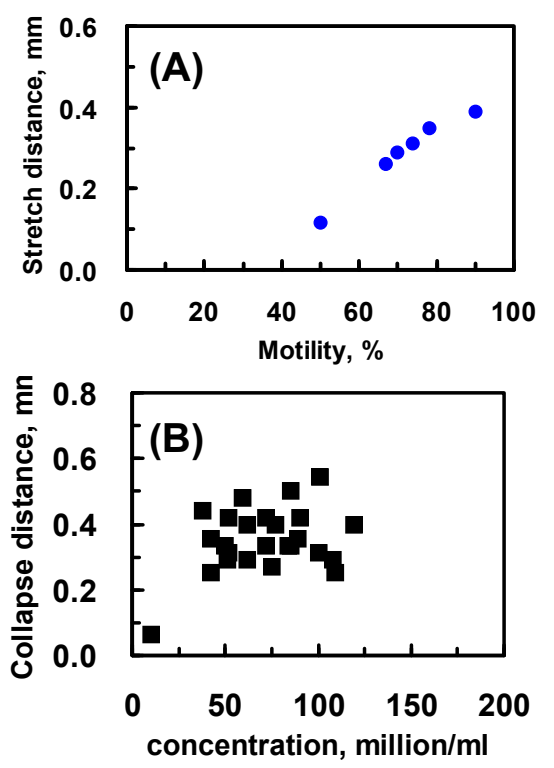

Figure 7: (A) The stretch distance of stretching semen drops as a function of motility. (B) The collapse distance of collapsing semen drops as a function of concentration.

$8 \mathrm{~F}$ and $8 \mathrm{G}$ ) of semen drops are also schematically depicted in Figures $8 \mathrm{~A}$ to $8 \mathrm{G}$. The semen drop simply consists of infertile sperms (gray sperms), fertile sperms (orange sperms), and seminal plasma (blue). In the beginning, the LC molecules anchored among the polymer grains are aligned along $y$-direction and all the sperms uniformly disperse in the semen drop (Figure 8A). When we apply the electric field at the left region of LCPCF, the LC molecules tilt up. The cyano terminal group of LC molecules is more hydrophilic; as a result, the left side of the LCPCF is more hydrophilic as well. The semen drop experiences a net Young's force resulting from the change of the wettability. A fluidic flow (or the flow of seminal plasma) inside the semen drop was then induced. (The white arrow in Figure 8B) All the sperms are then flushed by such a fluidic flow. However, the fertile sperms swim upstream against the fluidic flow because of the nature of the fertile sperms [17] (Figure $8 \mathrm{~B})$. When we turn off the voltage, the LC molecules reorientate back along $y$-direction owing to the elastically essential properties of $\mathrm{LC}$ and the anchoring force of polymer grains. The wettability of LCPCF goes back. The fertile and infertile sperms then dispersed inside the semen drop (Figure 8C). Therefore, we observe the back-and-forth stretch of a semen drop when the voltage is on and off periodically. The high concentration, high motility, better morphology, high grade $\mathrm{A}$, and low grade B represent that more sperms can swim upstream against the flow current of seminal plasma induced by the wettability gradient of LCPCF. The mechanism of the collapse of the semen drop is also illustrated in Figure 8D, 8E, 8F, and 8G. When the semen drop has lots of infertile sperms, the infertile sperms are washed away by the fluidic flow induced by the wettability gradient due to the orientation of LC molecules (Figure 8E). Because the infertile sperms have weak ability to swim against the flow, the infertile sperms are attracted and trapped by the field of liquid crystals and weak fringing electric fields on the surface of LCPCF (Figure 8F). The surface of LCPCF is then remodified. As a result, the switchable properties of LCPCF are invalid by the deposition of the sperms on LCPCF. Therefore, the collapse of semen drop and the aggregation near the voltage region in Figure 5 are observed (Figure 8G). Actually, some of the infertile sperms of the stretching semen drops were also trapped on the surface of LCPCF, similar to the collapsed semen drops. That is the reason why the contact angles of stretches of semen drops could not fully recover.

In conclusion, we demonstrated a sperm testing device on a basis on the motion of a semen drop on switchable surface of LCPCF. The better quality spermatozoa results in back-and-forth stretches of a semen drop on LCPCF; otherwise, the semen drop collapses. Such

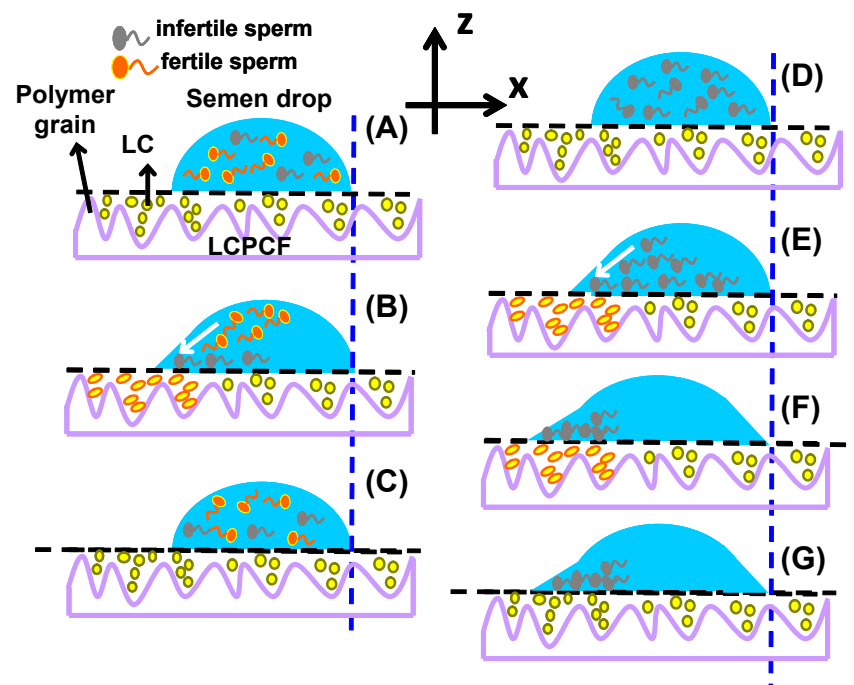

Figure 8: The schematic mechanism of stretches of a semen droplet. (A) At $V=0, L C$ molecules are aligned along y-direction. The fertile and infertile sperms swim randomly. (B) When the voltage is applied to the left of LCPCF the LC molecules tilt up and the left LCPCF is more hydrophilic. The induced fluidic flow (white arrow) washes away all the sperms meanwhile the fertile sperms swim upstream against the flow. (C) When the voltage turns off, the wettability of LCPCF is same as (A) and the semen drop stretches back. (D) At $V=0$, the infertile sperms disperse in the semen drop. (E) When the voltage is applied to the left region of LCPCF, the induced fluidic flow (white arrow) washes away all the sperms. (F)(G) The infertile sperms are deposited on the left region. 
Citation: Lin Y, Chu T, Chu W, Tsou Y, Chiu Y, et al. (2011) A Sperm Testing Device on a Liquid Crystal and Polymer Composite Film. J Nanomedic Nanotechnol S9:001. doi:10.4172/2157-7439.S9-001

Page 5 of 5

stretching of a semen droplet indicates the high concentration $(>100$ million/mL), good morphologies $(>15 \%)$, good motility $(>50 \%)$ of spermatozoa, and more than $30 \%$ of spermatozoa swimming jointly forward. The stretch distance and collapse distance also indicates the motility and concentration of semen, respectively. The mechanisms of back-and-forth stretches and collapses of semen drops are also discussed. We acknowledge the fact that fertilization involves many complicated sperm-oocyte processes and is not decided by sperm quality alone. However, good sperm quality is still a significant factor for fertility potential. Here, the experimental results indicate the great capabilities of LCPCF to be used as a sperm quality tester. Potential applications for this device include sperm testers and microfluidic devices for Assisted Reproductive Technology.

\section{Acknowledgements}

The authors are indebted to Benjamin Wu (Duke University) for proofreading the manuscript, and Liang-Chieh Peng and Pi-Yu Chen (Ton Yen General Hospital), Cheng-Yu Shen (NSYSU), Prof. Shu-Hsia Chen (NCTU), and Dr. YungHsun Wu (Chimei-Innolux Corp.) for useful discussions and technical assistance. This research was supported by National Science Council (NSC) in Taiwan under the contract No. 98-2112-M-009-017-MY3.

\section{References}

1. Daniel S, Chaudhury MK (2002) Rectified motion of liquid drops on gradient surfaces induced by vibration. Langmuir 18: 3404-3407.

2. Gras SL, Mahmud T, Rosengarten G, Mitchell A, Kalantar-zadeh K (2007) Intelligent control of surface hydrophobicity. Chemphyschem 8: 2036-2050.

3. Liu Y, Mu L, Liu B, Kong J (2005) Controlled switchable surface. Chem Eur J 11: 2622-2631.

4. Julthongpiput D, Lin YH, Teng J, Zubarev ER, Tsukruk VV (2003) Y-shaped amphiphilic brushes with switchable micellar surface structures. J Am Chem Soc 125: 15912-15921

5. Sun T, Wang G, Feng L, Liu B, Ma Y, et al. (2004) Reversible switching between superhydrophilicity and superhydrophobicity. Angew Chem Int Ed 43: 357-360.

6. Gallardo BS, Gupta VK, Eagerton FD, Jong LI, Craig VS, et al. (1999)
Electrochemical principles for active control of liquids on submillimeter scales. Science 283: 57-60.

7. Ichimura K, Oh SK, Nakagawa M (2000) Light-driven motion of liquids on a photoresponsive surface. Science 288: 1624-1626.

8. Ito Y, Heydari M, Hashimoto A, Konno T, Hirasawa A, et al. (2007) The movement of a water droplet on a gradient surface prepared by photodegradation. Langmuir 23: 1845-1850.

9. Feng CL, Zhang YJ, Jin J, Song YL, Xie LY, et al. (2001) Reversible wettability of photoresponsive fluorine-containing azobenzene polymer in LangmuirBlodgett films. Langmuir 17: 4593-4597.

10. Kim K, Jeon WS, Kang JK, Lee JW, Jon SY, et al. (2003) A pseudorotaxane on gold: formation of self-assembled monolayers, reversible dethreading and rethreading of the ring, and ion-gating behavior. Angew Chem 115: 2395-2398.

11. Lahann J, Mitragotri S, Tran TN, Kaido H, Sundaram J, et al. (2003) A reversibly switching surface. Science 299: 371-374.

12. Lin YH, Ren HW, Wu YH, Wu ST, Zhao Y, et al. (2008) Electrically tunable wettability of liquid crystal/polymer composite films. Opt Express 16: 17591 17598.

13. Ren H, Wu ST, Lin YH (2008) In situ observation of fringing-field-induced phase separation in a liquid-crystal-monomer mixture. Phys Rev Lett 100: 117801.

14. Lin YH, Li JK, Chu TY, Hsu HK (2010) A bistable polarizer-free electro-optical switch using a droplet manipulation on a liquid crystal and polymer composite film. Opt Express 18: 10104-10111.

15. De Gennes PG, Brochard-Wyart F, Quere D (2004) Capillarity and Wetting Phenomena Drops, Bubbles, Pearls, Waves. Springer-Verlag, Berlin.

16. De Gennes PG (1985) Wetting: statics and dynamics. Rev Mod Phys 57: $827-$ 863.

17. Seo D, Agca Y, Feng Z, Critser J (2007) Development of sorting, aligning and orienting motile sperm using microfluidic device operated by hydrostatic pressure. Microfluid Nanofluidics 3: 561-570.

18. World Health Organization (1999) WHO Laboratory Manual for the Examination of Human Semen and Sperm-Cervical Mucus Interaction. (4thedn), Cambridge University Press.

19. Jeyendran R (2003) Protocols for Semen Analysis in Clinical Diagnosis Parthenon Publishing Group. 